\title{
Detection of Depression Symptoms Using Chatbots Based on Machine Learning
}

\author{
Vitor Bastos \\ vitor_bss10@hotmail.com \\ CEFET/RJ-Campus Petrópolis
}

\author{
André Felipe Monteiro \\ andre.monteiro@cefet-rj.br \\ CEFET/RJ-Campus Petrópolis
}

\begin{abstract}
Nowadays depression is a relevant issue due the high level of stress observed even in students and young people. Moreover, the detection of the depression symptons is a complex task, since each person has different behaviors and reactions in these scenarios. This work address the detection of depression symptoms using chatbots based on machine learning algorithms. The use of chatbots enables a smooth approach for shy and introspective people, whose do not feel comfortable for talking to parents, psychologists or medical professionals in general. To this end, an App for smart-phone is proposed in order to perform a talk with a person, and verify if some depression symptoms are observed based using machine learning algorithms. The initial results show that the proposed model has a good accuracy on simulated scenarios, where basic talks are performed by the chatbot.
\end{abstract}

\section{KEYWORDS}

Chatbot, Aprendizado de máquina, Telemedicina

\section{INTRODUÇÃO}

Atualmente no Brasil, cerca de 5,8\% da população sofre de depressão, o que corresponde a 2 milhões de casos por ano conforme mostrado em [1]. A depressão ou qualquer outra doença psiquiátrica são ainda um tabu, mas representam uma ameaça relevante para a sociedade em virtude da ocorrência de suicídios e outras consequências graves para os envolvidos. $\mathrm{Na}$ maior parte dos casos a depressão não é diagnosticada, a falta de informação é principal motivo para que não haja procura por ajuda profissional. Se essas pessoas pudessem em um diálogo simples descobrir a doença em potencial, o acesso ao tratamento adequado seria ampliado.

É comum o uso de inteligência artificial no dia a dia, grandes empresas já proporcionam a interação homem e máquina de formas naturais em serviços de atendimento ao cliente, por exemplo, além de aplicações na área de saúde como descrito em [2] e [3]. A literatura apresenta diversas soluções baseadas em chatbots para auxiliar o tratamento de doenças psicossomáticas já diagnosticadas, conforme trabalhos descritos em [4] e [5]. Entretanto, a utilização de [? ] para auxiliar o processo de detecção de doenças psicossomáticas ainda é incipiente. Essa abordagem se apresenta como uma tendência promissora, em virtude de sua capilaridade e boa aceitação dos usuários, de acordo com [6].

Tendo em vista o cenário de utilização massiva de smartphones e aplicativos de conversação e redes sociais, prover uma ferramenta complementar para diagnóstico remoto de doenças psicossomáticas mostra-se uma abordagem relevante. Entretanto, um dos grandes desafios para o desenvolvimento de chatbots é conseguir manter o contato com o usuário, já que a falta de interesse dos usuários decorre da maneira não intuitiva que os chatbots implementam o processo de conversação conforme estudos recentes apresentados em [7], [8] e [9].

Este trabalho apresenta um chatbot para auxiliar o processo de identificação de sintomas de depressão e outras doenças psicossomáticas. O objetivo é usufruir das relações naturais e impessoais de um chatbot e identificar características que possam representar sintomas de depressão. Caso seja identificado qualquer possível relação com a depressão, o chatbot entra automaticamente em um diálogo com perguntas referentes à referida doença, e após algumas respostas apresenta o grau em que o usuário pode apresentar de depressão. Além da depressão, a solução proposta neste trabalho também conta com algumas interações relacionadas às doenças de Transtorno obsessivo-compulsivo (TOC ), Transtorno Bipolar, Anorexia, Transtorno dismórfico corporal (TDC ), Perturbação borderline da personalidade (PBP) ou transtorno da personalidade borderline (TPB), Esquizofrenia, Fobia Social, Depressão, Estresse Pós-traumático e Depressão Pós-parto.

Este trabalho encontra-se organizado da seguinte forma: $\mathrm{Na}$ Seção 2 apresentamos o modelo proposto. Na Seção 3 descrevemos os testes iniciais realizados para avaliação do chatbot proposto. Por fim, na Seção 4 são apresentadas as considerações finais.

\section{MODELO PROPOSTO}

Este projeto foi desenvolvido utilizando a plataforma DialogFlow, disponibilizada pela Google. A plataforma é capaz de prover interfaces conversacionais entre homem e máquina, e tem grande capacidade de entendimento de linguagem natural. O DialogFlow trabalha com conceitos de NLP (Natural Language Processing - Processamento de Linguagem Natural). A NLP é uma subcamada da Inteligência Artificial que tem como foco o entendimento natural da linguagem humana. Além de entender um texto, a plataforma é capaz de analisar e aprender como reconhecer o mesmo texto escrito de formas diferentes.

Utilizamos o conceito de intents, onde o processo de aprendizado de máquina identifica a frase do usuário e retorna uma resposta como for programado pelo intent. Assim, o mesmo intent pode ser integrado a uma API externa, por meio de um webhook, e então fornecer informações ou funções adicionais. Além disso, aplicamos o conceito de contexts, que determinam o contexto da conversa, para avaliar qual a resposta mais adequada ao usuário. Além dos intents e contexts, utilizamos as Entities, que são entidades programáveis como data, tempo, cor e outros. Neste projeto as Entities foram implementadas para entendimento dos sintomas de depressão e seus respectivos sinônimos pelo chatbot.

O Dialogflow tende a sempre fazer parse (combinação entre a pergunta do usuário e resposta do bot) com um intent, independente de o usuário estar efetuando os questionários propostos, e então interrompendo a coleta de dados. Os contexts são a solução para o 
problema, desde que que todas as interações sejam configuradas com seus respectivos contextos. Desta forma, é possível manter o usuário em uma série de interações, a não ser que o usuário solicite sua saída. Integrações exteriores facilitam o uso da ferramenta, que já proporciona de forma direta acesso ao Whatsapp, Facebook Messenger, Twitter, Skype, Telegram, dentre outros.

Apesar deste trabalho ainda estar em fase inicial de desenvolvimento, o mesmo já possui mais de 100 possíveis diálogos de uma conversa simples e identifica como gatilho para a doença da depressão cerca de 30 palavras chaves, e seus possíveis sinônimos, com expansão automática em função do processo de aprendizado de máquina utilizado. Após a identificação do gatilho, o usuário é submetido a um "questionário" rápido, que foi baseado na solução apresentada em em uma versão resumida do teste de depressão criado pelo Dr. Ivan Goldberg e disponibilizado em uma rede social independente de saúde mental a PsychCentral [10]. O questionário é composto por 19 perguntas rápidas, esperando respostas como Nenhum, Pouco, Moderado e Bastante. Na Fig. 1 apresentamos capturas de tela de uma conversa entre uma pessoa e o chatbot. Na tela da esquerda encontra-se o início da interação, e na direita tem o diagnóstico que o chatbot fez do usuário.
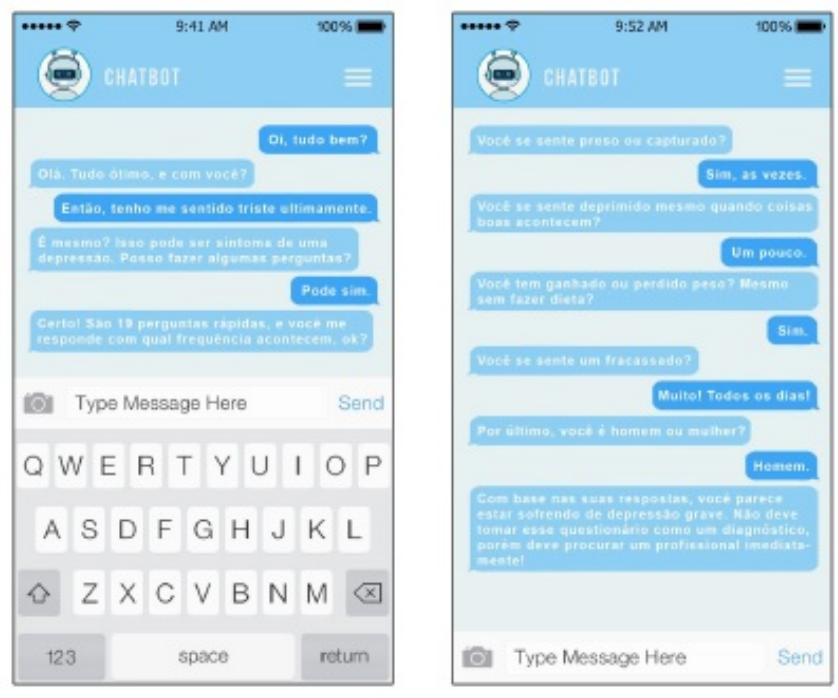

Figure 1: Exemplos de diálogos com o Chatbot

As perguntas foram modeladas para que o usuário possa responder com que frequência a situação ocorre, além de ser mais intuitivo, facilita no momento de dar o resultado. Foi adicionada uma valoração, tanto nas perguntas que podem ter peso de 1 a 10 , e nas respostas, com nível de 1 a 4 (nenhum, pouco, moderado e bastante). O resultado é dado por uma média ponderada entre as 18 (a 19 pergunta é sobre o sexo do usuário, e não se aplica ainda no resultado) perguntas e suas respostas, e a resposta do chatbot é programada com base em um score feito pelo usuário, que vai de $1 \mathrm{a}$ 4, além de ser arquivada em um banco de dados mySQL e acessado a qualquer momento pelo usuário. No ambiente de testes iniciais todas as perguntas foram definidas com a mesma valoração de 10 pontos, que seria o valor máximo. A pontuação final do usuário pode variar de 1 a 4, e é definida conforme a Eq. 1 apresentada a seguir, onde $V P_{i}$ é o valor associado à pergunta $i, R P_{i}$ é o valor correspondente à resposta $i$, e 18 é o total de perguntas realizadas ao usuário.

$$
\text { PontuaoFinal }=\sum_{i=1}^{18} \frac{V P_{i} \cdot R P_{i}}{V P_{i}}
$$

A pontuação final possui valores inteiros de 1 a 4, indicando ao usuário o diagnóstico obtido por meio do questionário. As mensagens apresentadas aos usuários associadas a cada valor de pontuação final são indicadas a seguir:

(1) "Você parece estar muito bem, mas é recomendado ratificar este diagnóstico junto a um profissional."

(2) "Verificamos que você apresenta alguns indícios relacionados a doenças psicossomáticas. Indicamos procurar um profissional para um diagnóstico mais detalhado."

(3) "Você apresenta diversos indícios relacionados a doenças psicossomáticas. Indicamos procurar um profissional o mais breve possível."

(4) "ALERTA! Você apresenta indícios muito elevados relacionados a doenças psicossomáticas. Indicamos procurar um profissional com urgência."

\section{RESULTADOS INICIAIS}

Para a avaliação de desempenho do modelo proposto, optamos por iniciar os testes avaliando a capacidade da solução proposta em identificar sinônimos e frases similares. Essas funcionalidades estão relacionadas à capacidade de generalização e adaptação do chatbot, visto que a interpretação de linguagem natural é um grande gargalo para uma melhor interação homem-máquina. Além disso, o processo de aprendizado de máquina deve ser capaz de desenvolver uma conversação intuitiva com o usuário, de forma que o mesmo sinta-se confortável em interagir com a ferramenta sem maiores receios

Foram efetuados testes iniciais para avaliação da capacidade de entendimento do modelo proposto. A Tabela 1 apresenta 6 frases com significado semelhante da frase "Tenho me sentido triste", e as taxas de acerto do chatbot na identificação de sinônimos. A submissão das frases foram de forma aleatória, e 20 vezes cada uma delas em momentos diferentes.

\begin{tabular}{l|c|c} 
Frase & $\mathbf{1}^{\boldsymbol{o}}$ teste & $\mathbf{2}^{\boldsymbol{o}}$ teste \\
\hline Tenho me sentido irritado & $100 \%$ & $90 \%$ \\
\hline Estou triste ultimamente & $100 \%$ & $100 \%$ \\
\hline Me sinto triste & $100 \%$ & $100 \%$ \\
\hline Sinto muita tristeza & $100 \%$ & $100 \%$ \\
\hline As vezes me sinto muito triste & $100 \%$ & $100 \%$ \\
\hline Estou entristecido & $100 \%$ & $100 \%$ \\
\hline
\end{tabular}

Table 1: Detecção de frases sinônimas pelo Chatbot

Além do teste anterior, foi efetuado outro teste com objetivo entender a capacidade do modelo de identificar sinônimos em apenas uma palavra da mesma frase. Assim, utilizamos como base a 
frase "Tenho me sentido triste" substituindo a palavra "triste" pelos sinônimos "infeliz", "descontente", "deprimido", "aborrecido" e "desalegre”, além de uma variação errada com a grafia "trsite”. Para este teste, o chatbot desenvolvido apresentou uma acurácia total de $94 \%$, o que se mostra um resultado promissor para prosseguirmos com o desenvolvimento do modelo proposto para avaliação em cenários mais complexo.

\section{CONSIDERAÇÕES FINAIS}

O aprendizado de máquina do DialogFlow se mostrou eficiente nos testes controlados realizados neste trabalho. Além da grande acurácia em frases sinônimas dentro de um mesmo contexto, o chatbot também apresentou bons resultados para sinônimos de palavras independentes em uma mesma frase, mostrando uma promissora capacidade de adaptação e generalização. Como trabalhos futuros, além do prosseguimento da pesquisa para expandir a generalização do modelo proposto para outros tipos de doenças de caráter psicossomático, pretendemos integrar o chatbot nas redes sociais, principalmente no Whatsapp, tornando-se uma ferramenta de fácil acesso e de grande escala. Desta forma, por meio de um número telefônico a ser definido qualquer usuário poderá enviar mensagens para o chatbot para iniciar uma conversa e responder o questionário de avaliação mencionado anteriormente.

Por fim, cabe ressaltar que testes mais complexos serão efetuados, e profissionais na área da psicologia e psiquiatria serão inseridos no projeto para adequar a ferramente às premissas éticas e técnicas necessárias na área da medicina, além de contribuírem para o melhoramento do conteúdo da solução proposta.

\section{AGRADECIMENTOS}

Os autores agradecem ao CEFET/RJ pela bolsa PIBIC disponibilizada para este projeto.

\section{REFERÊNCIAS}

[1] Patricia Kozuchovski Daré and Sandra Noemi Caponi. Cuidado ao indivíduo com depressão na atenção primária em saúde. ECOS-Estudos Contemporâneos da Subjetividade, 7(1):12-24, 2017.

[2] Rafael A Calvo, David N Milne, M Sazzad Hussain, and Helen Christensen. Natural language processing in mental health applications using non-clinical texts. Natural Language Engineering, 23(5):649-685, 2017.

[3] AM Rahman, Abdullah Al Mamun, and Alma Islam. Programming challenges of chatbot: Current and future prospective. In 2017 IEEE Region 10 Humanitarian Technology Conference (R10-HTC), pages 75-78. IEEE, 2017.

[4] Pratik Kataria, Kiran Rode, Akshay Jain, Prachi Dwivedi, Sukhada Bhingarkar, and MCP India. User adaptive chatbot for mitigating depression. International fournal of Pure and Applied Mathematics, 118(16):349-361, 2018.

[5] Minha Lee, Sander Ackermans, Nena van As, Hanwen Chang, Enzo Lucas, and Wijnand IJsselsteijn. Caring for vincent: A chatbot for self-compassion. In Proceedings of the 2019 CHI Conference on Human Factors in Computing Systems, pages $1-13,2019$.

[6] Eduardo Vinicius Neves Biazotto and Ronaldo César Dametto. Chatbot: Engajamento do consumidor através da inteligência artificial.

[7] Alan O Santana and Eduardo HS Aranha. Um modelo de chatbot para aulas de desenvolvimento de jogos digitais. Anais do Computer on the Beach, pages 422-431, 2018.

[8] Vinicius Dalla Corte, Vagner Kaefer Dos Santos, and Dalcimar Casanova. Chatbot baseado em rede neural long short-term memory (lstm): um estudo de caso baseado no livro william shakespeare. Anais do Computer on the Beach, pages 484-492, 2019.

[9] Bayan AbuShawar and Eric Atwell. Alice chatbot: Trials and outputs. Computación y Sistemas, 19(4):625-632, 2015.

[10] Dr. Ivan Goldberg. Psych central. URL http://www.https://psychcentral.com. 